\title{
Laparoscopic guided local injection in the $X$-linked muscular dystrophy mouse (mdx) diaphragm. An advance in experimental therapies for Duchenne Muscular Dystrophy ${ }^{1}$
}

\author{
Thais Borges Lessa ${ }^{\mathrm{I}}$, Rafael Cardoso Carvalho" ${ }^{\mathrm{II}}$, Júlio David Spagnolo ${ }^{\mathrm{III}}$, Luis Claudio Lopes Correia da Silva ${ }^{\mathrm{IV}}$, Silvia Renata \\ Gaido Cortopassiv, Carlos Eduardo Ambrósiovi
}

DOI: http://dx.doi.org/10.1590/S0102-86502014001800004

IFellow PhD degree, Postgraduate Program in Anatomy of Domestic and Wild Animals, University of Sao Paulo (USP), Brazil. Intellectual and scientific content of the study, design the protocol, technical procedures, acquisition and interpretation of data, statistical analysis, manuscript writing.

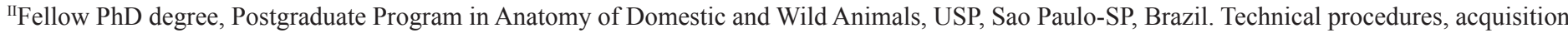
and interpretation of data.

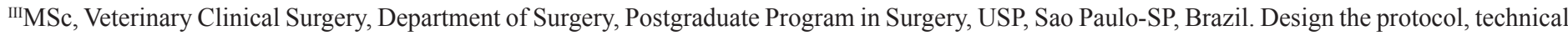
procedures.

${ }^{\text {IV }} \mathrm{PhD}$, Associate Professor, Department of Surgery, Veterinary School, USP, Sao Paulo-SP, Brazil. Design the protocol, technical procedures, acquisition of data, manuscript writing, critical revision.

vAssociate Professor, Department of Surgery, Veterinary School, USP, Sao Paulo-SP, Brazil. Design the anesthetic protocol, technical procedures.

${ }^{V I}$ Associate Professor, Department of Veterinary Medicine, Veterinary School, Faculty of Animal Sciences and Food, Engineering, USP, PirassunungaSP, Brazil. Intellectual and scientific content of the study, interpretation of data, manuscript writing, critical revision.

\section{ABSTRACT}

PURPOSE: To investigate the development of a laparoscopy technique for local injection into the X-linked muscular dystrophy (mdx) diaphragm.

METHODS: It was used 10 mice Balb/C57 and $5 \mathrm{mdx}$ mice and three differents decubitus type were tested: the right lateral, supine, and supine decubitus with 20 degrees elevation of the forelimb. Abdominal caudal face and the 10 intercostal space were tested as spot to introduce the needle into the diaphragm.

RESULTS: Supine position with elevation of 20 degrees forelimb and the 10th intercostal space are the beneficial position to apply a local injection.

CONCLUSION: It was proved to be possible to perform the laparoscopy technique in the X-linked muscular dystrophy diaphragm and this requires a specific position and technique during the surgery.

Key words: Diaphragm. Injections. Muscular Dystrophy, Duchenne. Laparoscopy. Mice. 


\section{Introduction}

Laparoscopic techniques are a minimally procedures, aiming to reduce the risks associated with conventional surgery, that proved superior to postoperative pain and recovery ${ }^{1}$.

Duchenne muscular dystrophy (DMD) is the most devastating X-linked muscle disorders that affect 1 in 3500 male's births, caused by mutation in gene encoding the $427 \mathrm{kDA}$ protein, called dystrophin ${ }^{2,3}$. The major respiratory symptoms observed in these patients, occurs, when they used the wheelchair. During this period, these patients are affected by scoliosis structural deformities, mainly responsible for the lung function decrease. Due the respiratory muscles weakness and structural deformities, it can observed the hipercarpnia, pulmonary hypoventilation, hypoxemia (secondary hipercarpnia) and therefore, secretion clearance, decreased lung compliance and chest mobility, increasing the mechanical loading sustained every respiratory movement by the weak musculature ${ }^{4}$.

Considering these respiratory complications, several experimental therapies are being performed to improve the respiratory conditions DMD patients ${ }^{5-7}$, evidencing the importance of developing new techniques and advances to resources for experimental therapies, e.g pharmacological and stem cells. Since it extend the resources of DMD respiratory muscles intervention, the chances of these patients maintain their activities of daily living without the use of noninvasive ventilatory resources and patient survival increases.

The $m d x$ (X- linked muscular dystrophy mouse) is an animal model commonly used to DMD studies. These animal can be easily and reliably reproduced and due to a spontaneous mutation in the dystrophin gene and lack of dystrophin expression in muscle tissues ${ }^{8,9}$. In the $m d x$, the limb skeletal muscle exhibits an initial periods of severe necrosis at 3-4weeks of age lifespan, however the limb muscles of $m d x$ mice undergo sufficient muscle fiber regeneration to compensate for the continued injury to fibers and muscle fiber degeneration. Although the diaphragm muscle exhibits progressive deterioration, similar to human $\mathrm{DMD}^{5,10,11}$.

Considering the $m d x$ features cited by the authors above, the benefits of laparoscopy and the possibility to expand access techniques, for respiratory muscles in patients with DMD, this study, aimed to develop a viable a $m d x$ laparoscopy technique, that allowed the protocol development for an injection spot in the diaphragm muscle of this clinical model.

\section{Methods}

This research was certified by the Ethical Principles in Animal Research adopted by "Ethic Committee in the use of animals" of the School of Veterinary Medicine and Animal Science of University of Sao Paulo, protocol number 2045/2010.

For this research, we used 10 mice Balb/C57 of the Vivarium of Pathology, School of Veterinary Medicine Animal Science, University of Sao Paulo and $5 \mathrm{mdx}$ mice the Vivarium of the ABC School of Medicine.

\section{Laparoscopy}

To perform the laparoscopic procedure and the establishment of a favorable decubitus for the diaphragm muscle viewing, were tested three types of position: the right lateral decubitus, supine and supine with 20 degrees of elevation of the forelimb. In all positioning tested were used the same anesthetic protocol and laparoscopic.

\section{Anesthetic protocol}

The animals were anesthetic with the combination of $50 \mathrm{mg} / \mathrm{Kg}$ ketamine (Ketamin- $\mathrm{S}^{\circledR}$, Cristália) and $2 \mathrm{mg} / \mathrm{kg}$ xylazine (Calmiun $^{\circledR}$, Agener União), remained the animals in the holding spontaneous breath.

\section{Laparoscopic protocol}

After anesthesia, was realized an incision of $0.5 \mathrm{~cm}$ in the lower abdomen at median sagittal plane for laparoscopic input. The $2,7 \mathrm{~mm}$ optic was introduced and stabilized with circular sutures with wire of 4-0 poligalactina. The pneumoperitoneum was preceded to the pressure of $2 \mathrm{mmHg}$ through a $25 \mathrm{X} 7 \mathrm{G}$ needle placed parallel the optical imput.

\section{Protocol for diaphragm muscle injection}

Two protocols were tested to define an effective perform to the local injection in the diaphragm muscle

\section{Protocol 1}

The injection in the diaphragm costal face was performed in nine animals Balb/C57 being used three animals in each decubitus cited above. In this protocol, the needle was introduced into the right antimere of the abdomen caudal face, near the midline. We used a needle with $24 \mathrm{G}$ caliber plugged into a disposable insulin syringe containing $0.2 \mathrm{ml}$ of Tryplan Blue. When it was introduced, the needle bevel was directed to the diaphragm caudal face, in order to chart a cranial path and upward (Figure 1, Protocol 1). 


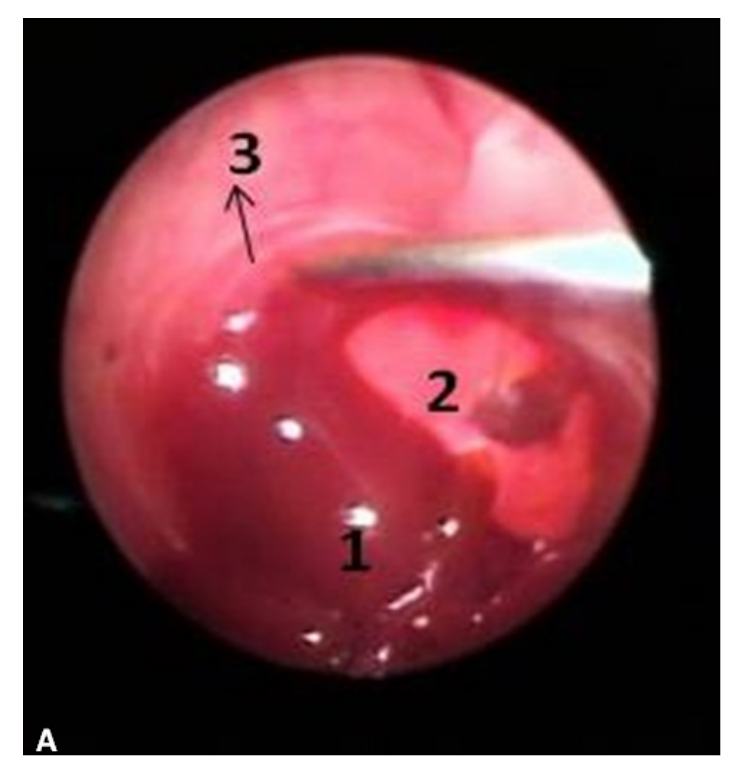

FIGURE 1 - Protocol 1. Laparoscopy image; caudal view of the diaphragm muscle. Observe the needle placed into the diaphragm costal face, (1) liver, (2) lung and (3) diaphragm costal face.

Protocol 2

The protocol 2 was utilized after the following criteria: the determination of the most favorable position for surgery (data obtained using protocol 1) and after checking the effectiveness of the protocol 1.

The protocol 2 was realized in supine position with elevation of 20 degrees of the thoracic limbs in $1 \mathrm{Balb} / \mathrm{C} 57$ and in $5 \mathrm{mdx}$. It was used a needle with $24 \mathrm{G}$ caliber plugged into the disposable insulin syringe with $0.2 \mathrm{ml}$ de Tryplan Blue. This one was introduced in the 10 intercostal space, piercing the intercostal muscle and the place where the diaphragm is inserted. When the bevel of the needle was visualized on the monitor, this was retreated until the entire needle orifice stay completely within the muscle, occupying a portion of the diaphragm and another in intercostal muscle (Figure 2, Protocol 2).

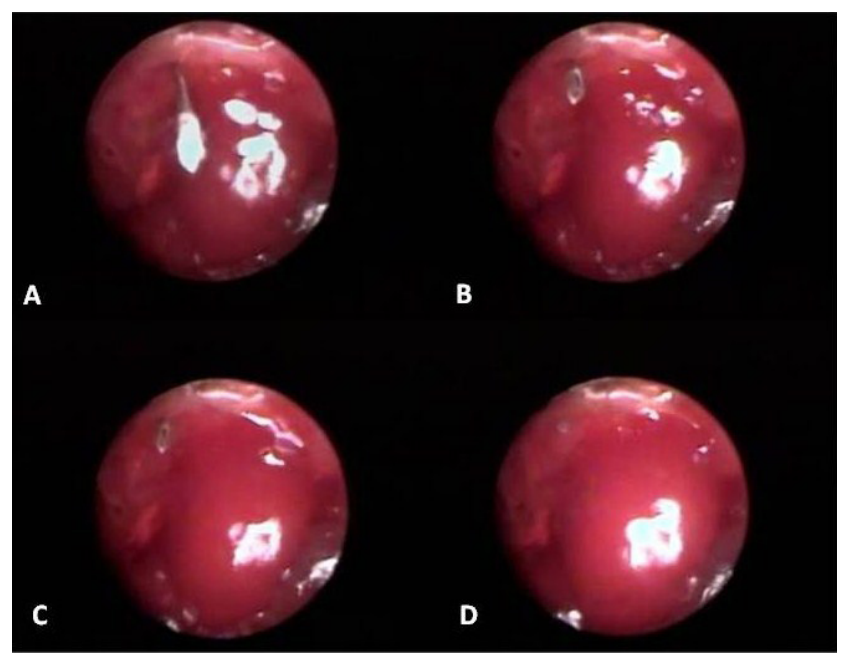

FIGURE 2 - Protocol 2. Laparoscopy image; caudal view of the diaphragm muscle. (A) Observe the needle into the abdominal cavity, which was introduced in the tenth intercostal space; (B and C) needle being retracted; (D) needle positioned between the diaphragm and intercostal muscle.

\section{Postoperative protocol}

The postoperative protocol was performed with 2.5 / $\mathrm{kg}$ flunixin meglumine for three days, and $2 \mathrm{mg} / \mathrm{kg}$ of meperidine hydrochloride three times daily during five days for analgesia. For prophylactic antibiotics were used $10 \mathrm{mg} / \mathrm{kg}$ of enrofloxacin by intramuscular injection two times per day. After 10 days, all animals that remained alive were euthanized with an overdose of anesthesia and diaphragmatic muscle was dissected.

\section{Results}

\section{Protocol 1 - Laparoscopy in supine position}

It was observed in this position, the liver of the animal confined large part of diaphragm costal face, hindering the injection spot. After the needle was introduced, we can observe the occurrence of pneumothorax and after, the death of the animal.

At the end of the procedure, the diaphragm was dissected for analysis. The dissection proceeded in antimeres with the removal of the integument and muscles of the chest and abdominal regions, and thus accessed their organs. For visualization of the diaphragm muscle, the abdominal and thoracic organs were removed. It was observed the diaphragm muscle emerging bundles and peripheral ligaments oriented radially, joining the central tendon presenting planed format. The tendons peripherals were connected ventrally in the sternum and at the xiphoid process, laterally to the ribs ( 7 th, 8 th, 9 th and 10 th, and apices of the 11 th and 12 th) and the correspondents costal cartilages and dorsally three lumbar vertebrae. It was noted the foramen of the vena cava, esophageal hiatus, aortic hiatus and your three divisions: sternal part, and costal part and the left and right pillars of the diaphragm muscle (Figure 3). 

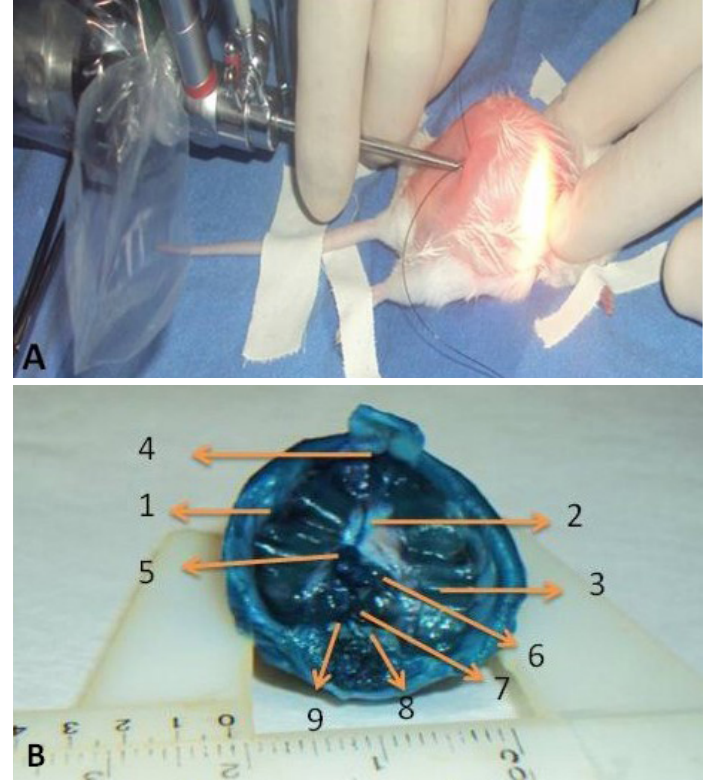

FIGURE 3 - (A) Balb/C57 photograph in supine position, we observe the optical with " $X$ " suture. (B) Caudal view of the diaphragm, (1) right costal face, (2) central tendon, (3) left costal face, (4) xiphoid process, (5) foramen vena cava, (6) esophageal hiatus, (7) aortic hiatus, (8) and left diaphragmatic pillars (9) right diaphragmatic pillars. Note the diaphragmatic muscle stained in blue, due to leakage of the Tryplan Blue.

\section{Protocol 1 - Laparoscopy in right lateral decubitus}

It was observed in this position, the animal's liver displacement of the diaphragm caudal face and the liver remained hindering the injection spot, but not precluding the injection. After the needle is inserted, we can observe the occurrence of pneumothorax and after, the death of the animal (Figure 4).
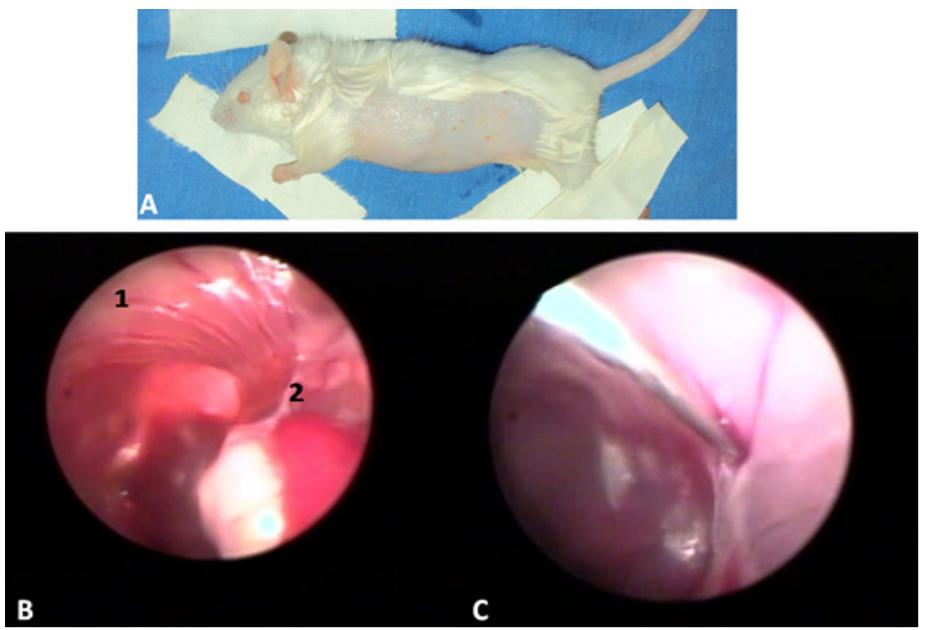

FIGURE 4 - (A) Balb/C57 photograph in right lateral decubitus. (B) Laparoscopy image, (1) left costal face and (2) ligament gastrophrenic of the stomach. (C) Injection spot.

At the end of the procedure, the diaphragm was dissected for analyses and it was observe the same results describe above.
Protocol 1 - Laparoscopy in supine position with 20 degrees of the thoracic limbs

During protocol 1, that the supine position with 20 degrees of thoracic limbs was favorable to visualize the diaphragm muscle, because the liver of the animal had a considerable displacement of the diaphragm costal face, facilitating the injection application. After the needle was introduced we can observe the occurrence of pneumothorax and after, the death of the animal. We observed that this decubitus was the most favored to perform the injection spot in the diaphragm. However this protocol has not kept the animals alive.

At the end of the procedure, the diaphragm was dissected for analyses and it was observe the same results describe above.

\section{Protocol 2 - Laparoscopy in supine position with 20 degrees of thoracic limbs}

During perform, of the protocol 2, after the needle be introduced, we can observe that there was no extravasation of Tryplan Blue in the abdominal cavity; pneumothorax has not occurred and the animals remained alive after the procedure. These animals were kept alive for 10 days and after all the animals were euthanized and the diaphragm muscle was dissected. The same morphological structures mentioned above were observed and it was verified that there was no rupture in the diaphragm muscle (Figure 5).

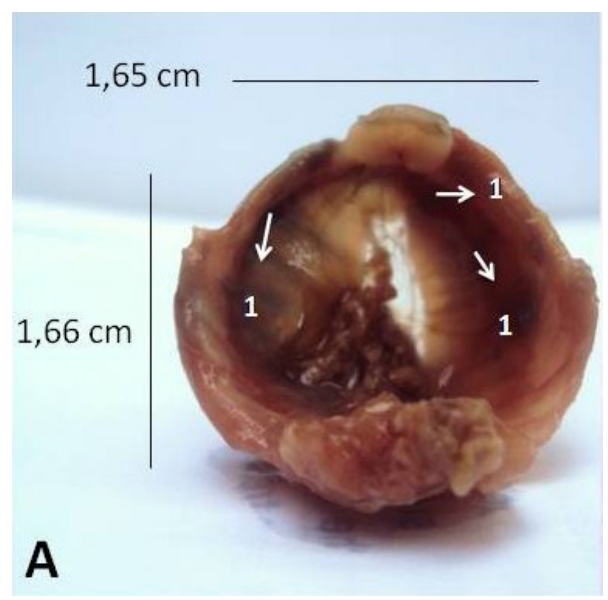

FIGURE 5-Macroscopic photography, caudal view of the diaphragm muscle. Observe the injection spot marked by Trypan Blue.

\section{Discussion}

In DMD, the Golden Retriever Muscular Dystrophy (GRMD) has been the most extensively animal model examined and characterized for elucidation of the pathogenesis and assessment 
of efficacy and toxicity, during the development of therapies ${ }^{3}$. Although the GRMD are a potential model of human disease, these models require a high maintenance cost and treatment, because they are large, which hinders their handling when was used in experiments. Unlike the $m d x$ model that presents easy reproduction, handling, low cost, low emotional involvement with his treater and vice versa, and convenience for experiments in laboratories ${ }^{8,9}$.

In recent years, the diaphragm muscle has been widely approached in the DMD studies ${ }^{5-7}$ which demonstrate the concern of researchers in obtaining data about this muscle.

The laparoscopic procedure has been successfully used in interventions in the diaphragm ${ }^{12,13}$. However, until the present moment there are no studies using laparoscopic procedure for local injection in the $m d x$ diaphragm.

Matecki et al. ${ }^{12}$, used the laparoscopy to expose the abdominal surface of the right hemidiaphragm in the $m d x$ and wild-type (C57BL10) and induce a massive necrosis with a topical application of notexin. After surgery, the authors reported that there were no complications, confirming the $m d x$ tolerance for this type of intervention. Despite, the local diaphragm access was described above, in this research, the size of the incision and the fact that there was not diaphragm perforation, differs these two techniques. In the laparotomy, the incision is larger than in laparoscopy, a fact that favors the laparoscopic technique for model $m d x$.

Regarding the parameters used in laparoscopy ${ }^{14}$, to develop a model of splenectomy in rats, used to establish pneumoperitoneum pressure of $7 \mathrm{mmHg}$ and introduced a $5 \mathrm{~mm}$ trocar. In one study to practice bariatric surgery in rats, the authors used the pressure of $8 \mathrm{mmHg}$ and $11 \mathrm{~mm}$ trocar. Meyer et al. ${ }^{16}$ to establish a model of partial nephrectomy, used 20 Wistar rats and established pneumoritônio with pressure of $5 \mathrm{~mm} \mathrm{Hg}$ and a $5 \mathrm{~mm}$ trocar. Ettinger et al. ${ }^{15}$, as well as the Meyer et al..$^{16}$, used a pressure of $5 \mathrm{mmHg}$ for establishing pneumoperitoneum. However, in our experiment, we used a $2.7-\mathrm{mm}$ of trocar size, because the animals used, has a smaller size and weight than the animals used in Meyer et al. ${ }^{16}$ and Ettinger et al. ${ }^{15}$.

\section{Conclusion}

It was proved to be possible to perform the laparoscopy technique in the X-linked muscular dystrophy diaphragm and this requires a specific position and technique during the surgery.

\section{References}

1. Liem MSL. Comparison of conventional anterior surgery and laparoscopic surgery for inguinal-hernia repair. N Engl J Med. 1997
May;336(22):1541-7. doi: 10.1056/NEJM199705293362201.

2. Spencer MJ, Tidball JG. Do immune cells promote the pathology of dystrophin-deficient myopathies? Neuromuscul Disord. 2001 Sep;11(6-7):556-64. PMID: 11525885.

3. Nakamura A, Takeda S. Mammalian models of Duchenne Muscular Dystrophy: pathological characteristics and therapeutic applications. J Biomed Biotechnol. 2011;2011:184393. doi: $10.1155 / 2011 / 184393$

4. Araujo APQC, Deco MC DE, Klôh BS, Costa MR, Góis FV, Guimarães AFCM. Diagnosis delay of duchenne muscular dystrophy. Rev Bras Saude Mater Infant. 2004 Jun;4(5):179-83. doi. org/10.1590/S1519-38292004000200008.

5. Gayraud J, Matecki S, Hnia K, Mornet D, Prefaut C, Mercier J, Michel A, Ramonatxo M. Ventilation during air breathing and in response to hypercapnia in 5 and 16 month-old $m d x$ and C57 mice. J Muscle Res Cell Motil. 2007 Apr;28(1):29-37. PMID: 17431804.

6. Rafael-Fortney JA, Chimanji NS, Schill KE, Martin CD, Murray JD, Gangul YR, Stangland JE, Tran T, Xu Y, Canan BD, Mays TA, Delfín DA, Janssen PM, raman SV. Early treatment with lisinopril and spironolactone preserves cardiac and skeletal muscle in Duchenne muscular dystrophy mice. Circulation. 2011 Apr;124(5):582-8. doi: 10.1161/CIRCULATIONAHA.111.031716.

7. Huang P, Cheng G, Lu H, Aronica M, Ransohoff RM, Zhou L. Impaired respiratory function in $m d x$ and $m d x / u t r n+/-$ mice. Muscle Nerve. 2011 Feb;43(2):263-7. doi: 10.1002/mus.21848.

8. Lynch GS, Hinkle RT, Faulkner JA. Force and power output of diaphragm muscle strips from $m d x$ and control mice after clenbuterol treatment. Neuromuscul Disord. 2001 Mar;11(2):192-6. PMID: 11257477.

9. Gosselin LE, Barkley JE, Spencer MJ, McCormick KM, Farkas GA. Ventilatory dysfunction in $m d x$ mice: impact of tumor necrosis factor-alpha deletion. Muscle Nerve. 2003 Sep;28(3):336-43. doi: 10.1002/mus. 10431 .

10. Hartel JV, Granchelli JA, Hudecki MS, Pollina CM, Gosselin LE. Impact of prednisone on TGF- $\beta 1$ and collagen in diaphragm muscle from $m d x$ mice. Muscle Nerve. 2001 Mar;24(3):428-32. PIMD: 11353432

11. Yeung EW, Whitehead NP, Suchyna TM, Gottlieb PA, Sachs F, Allen DG. Effects of stretch-activated channel blockers on [Ca2+] $\mathrm{i}$ and muscle damage in the $m d x$ mouse. J Physiol. 2005;562(Pt 2):367-80.

12. Matecki S, Guibinga GH, Petrof BJ. Regenerative capacity of the dystrophic $(m d x)$ diaphragm after induced injury. Am J Physiol Regul Integr Comp Physiol. 2004 Jan;87(4):R961-8. doi: 10.1113/ jphysiol.2004.075275.

13. Matz A, Landau O, Alis M, Charuzi I, Kyzer SL. The role of laparoscopy in the diagnosis and treatment of missed diaphragmatic ruptures. Surg Endosc. 2000 Jun;14(6):537-9. PMID: 10890960.

14. Costa VA, Oliveira FM, JR Oliveira LC, Carreiro MC, Guimarães P. Modelo experimental de esplenectomia laparoscópica em ratos. Act Cir Bras. 2003 Jun;18(6):553-6. doi: 10.1590/S010286502003000600013.

15. Ettinger JE, Santos-Filho PV, Oliveira PD, Ázaro E, Mello CAB, Amaral PCG, Fahel E. Laparoscopic gastric banding in the rat model as a means of videolaparoscopic training. Obes Surg. 2006 Jul;16(7):903-7. PMID: 16839491.

16. Meyer F, Ioshii SO, Shin EWK, Esser DM, Marcondes RT, Patriani RH, Pimpão BF. Nefrectomia parcial laparoscópica em ratos. Acta Cir Bras. 2007 Feb;22(2):152-6. doi: 10.1590/S010286502007000200014 . 
Lessa TB et al.

\section{Correspondence:}

Carlos Eduardo Ambrósio

Avenida Duque de Caxias Norte, 225

13635-900 Pirassununga - São Paulo Brasil

Tel.: (55 19)3565-6871

ceambrosio@usp.br

Received: June 12, 2014

Review: Aug 14, 2014

Accepted: Sep 15, 2014

Conflict of interest: none

Financial sources: Sao Paulo Research Foundation (FAPESP), Coordination of Improvement for Higher Academic Staff (CAPES)

${ }^{1}$ Research performed at Faculty of Veterinary Medicine and Animal Science, Department of Surgery, University of Sao Paulo (USP), Brazil. Part of Master degree thesis, Postgraduate Program in Anatomy of Domestic and Wild Animals. Tutor: Prof. Dr. Carlos Eduardo Ambrósio. 